\title{
The Image Generating Algorithms of Micro-pits with Direction-uniformity Distribution of YAG Laser Texturing Roll Based on Square Partial Scrambling
}

\author{
Hongcai Wang ${ }^{1,2, a}$ and Yang Wang ${ }^{1, b}$ \\ ${ }^{1}$ School of Mechatronics Engineering, Harbin Institute of Technology, Harbin, Heilongjiang, 150001, \\ China \\ ${ }^{2}$ Key Laboratory of Mechanics in Advanced Manufacturing, Institute of Mechanics, Chinese \\ Academy of Sciences, Beijing, 100190, China \\ ahcwang@imech.ac.cn, bwyyh@hit.edu.cn
}

Keywords: Surface roughness, Evaluation, Laser texturing, Image generating algorithm, Uniformity.

\begin{abstract}
Two image generating algorithms based on square partial scrambling are raised to implement micro-pits with approximate uniformity distribution of YAG laser texturing roll. Micro-pits can be uniformly distributed to ensure the area-uniformity of the micro-pits distribution. Micro-pits can be partial scrambled and optimized according to the evaluation standard to ensure the direction-uniformity of the micro-pits distribution.Partial scrambling image generating algorithms based on square-aligned-array arrangement and square-staggered-array arrangement are both effective image generating algorithms of uniformity distribution. The latter is more effective.
\end{abstract}

\section{Introduction}

Texturing cold-rolled sheet is leveled and copied by texturing rolls. It has excellent stamping performances and coating properties. So it is an important raw material in industries of the automobile, household appliances, etc. Roll texturing technology is a technology which can make burnished roll textured, so the surface of the roll has special surface morphology. Presently, shot blast texturing(SBT), electrical discharge texturing(EDT) and laser texturing(LT) are roll texturing technologies which have been large-scale promoted in industry[1].

According to the classification of laser type, there are two types of laser texturing. They are $\mathrm{CO}_{2}$ laser texturing and YAG laser texturing. The YAG Laser texturing system via special Q-Switch modulation outputs high energy density, high repetition rate YAG laser pulses, and focuses them on the surface of a revolving roll to generate on the roll surface large amounts of molten pools with obvious craters, which are produced by an auxiliary gas stream side-blowing at a certain angle with preset ingredients and preset pressure. After the laser treatment, the roll surface is quickly hardened due to rapid cooling of the molten pools via heat conduction. Thus, the roll surface exhibits a definite morphology with large amount of hardened micro-pits surrounded by craters. When the laser pulses move along the roll axis with a constant speed, large amount of phase change hardened micro-pits will be formed on the roll surface. Afterward when the roll is used in thin plate rolling or temper rolling, these hardened craters will produce deformed and hardened micro-pits on the surface of the sheet under rolling, and laser texturing cold-rolled sheets are thus produced[2].

The micro-pits on the surface of YAG texturing roll are uniform in size and isolated from each other. Their density is precise and controllable. Their distribution is one-dimensional controllable and regular. Because the micro-pits have special surface morphology, LT texturing sheet has better stamping performances and coating properties. So it has higher quality than SBT texturing sheet and EDT texturing sheet[3]. But the percentage elongation in different direction on LT texturing sheet is different because the distribution of the micro-pits are not isotropic. Regular distribution of micro-pits produces visual difference at different direction and moire fringe easily[2]. 
The ideal distribution form of micro-pits on texturing roll is approximate isotropic and irregular distribution. That is, the distribution of height amplitudes are approximate same in any area and any direction on the texturing roll surface. Image engraving technology can grave image to the roll directly, so it is significant for improving performances of YAG laser texturing sheet to study the image generating algorithms of approximate direction-uniformity disordered distribution[2].

The document 4 comes up with height amplitude parameters of surface roughness based on area-direction character and their assessed index. Area-directional surface arithmetic mean deviation $S R a$ and its coefficient of variation $S R a_{d c v}$ can be used to assess the directional uniformity of the distribution situation of height amplitudes in a single assessed area[4].

\section{Partial scrambling image generation algorithm based on square-aligned-array arrangement}

Figure 1 is the planform of a micro-pit. Figure 2 is the cross section of a micro-pit. Each pixel represents the area of a square of $2 \times 2 \mu \mathrm{m}^{2}$. The gray value of the pixel represents the mean value of the height of the area. Each gray level represents the height of $0.1 \mu \mathrm{m}$. The diameter of the micro-pit is $150 \mu \mathrm{m}$. The bulgy height of the micro-pit is $5 \mu \mathrm{m}$. The depth of the micro-pit is $20 \mu \mathrm{m}$.

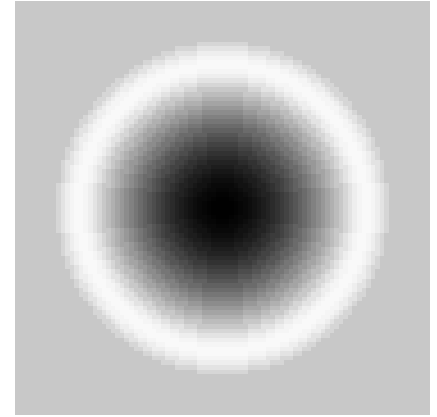

Fig. 1 The planform of a micro-pit

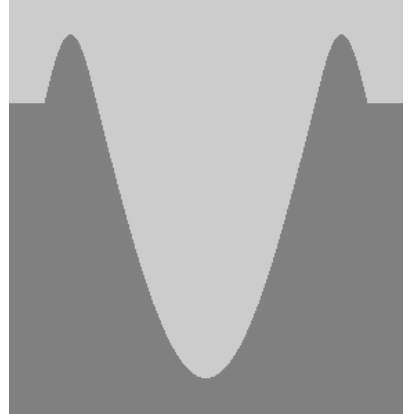

Fig. 2 The cross section of a micro-pit

Regular micro-pits image. $14 \times 14$ micro-pits are aligned by square array on the image which size is $3 \times 3 \mathrm{~mm}^{2}$. The distance of the pits is $200 \mu \mathrm{m}$. They are placed in the middle. Figure 3 is the generated regular micro-pits image.

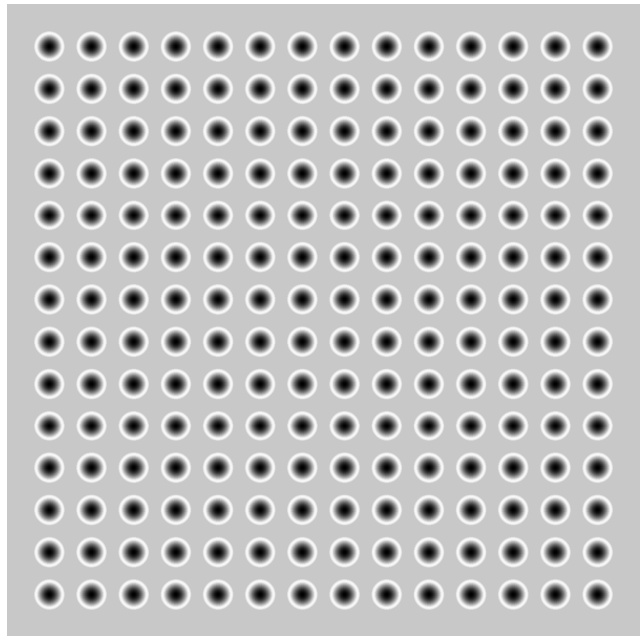

Fig. 3 Regular micro-pits image based on square-aligned-array arrangement

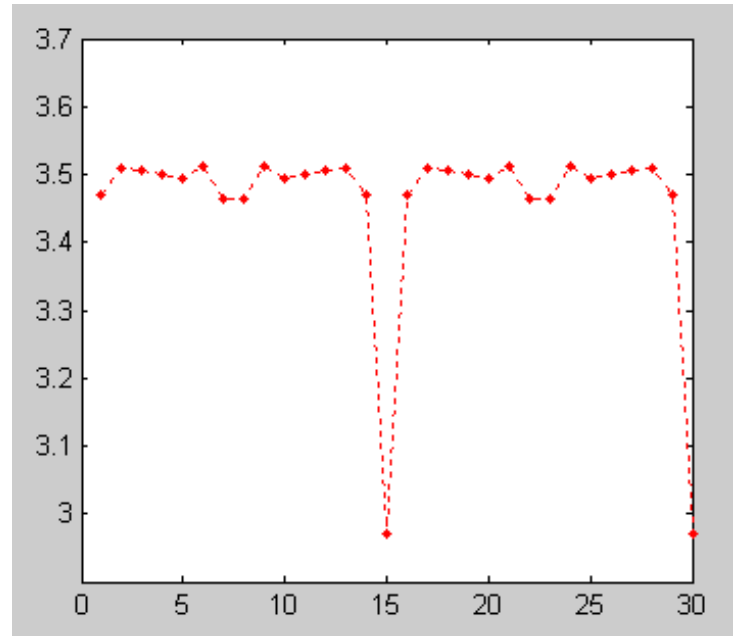

Fig. $4 S R a$ of regular micro-pits image based on square-aligned-array arrangement

The assessed area is a circle in the middle. The diameter of the circle is $2.35 \mathrm{~mm}$. Figure 4 is $S R a$ of regular micro-pits image based on square-aligned-array arrangement, in which vertical coordinate represents $S R a$, and the measurement unit is $\mu \mathrm{m}$, while horizontal coordinate represents direction value, each value represents 6 degrees. The results are: $S R a=2.97 \sim 3.51 \mu \mathrm{m}, S R a_{d c v}=3.80 \%$. 
Optimize the micro-pits image. Each micro-pit can be changed in the area of a $200 \times 200 \mu \mathrm{m}^{2}$ square area to improve the direction-uniformity of micro-pits distribution and ensure the micro-pits do not overlap each other. To obtain the micro-pits image of approximate direction-uniformity distribution, LDSE(low dimensional simplex evolution algorithms) advanced by Changtong Luo is adopted[5], and his software LDSE_Algorithm v3.1.8 is used.

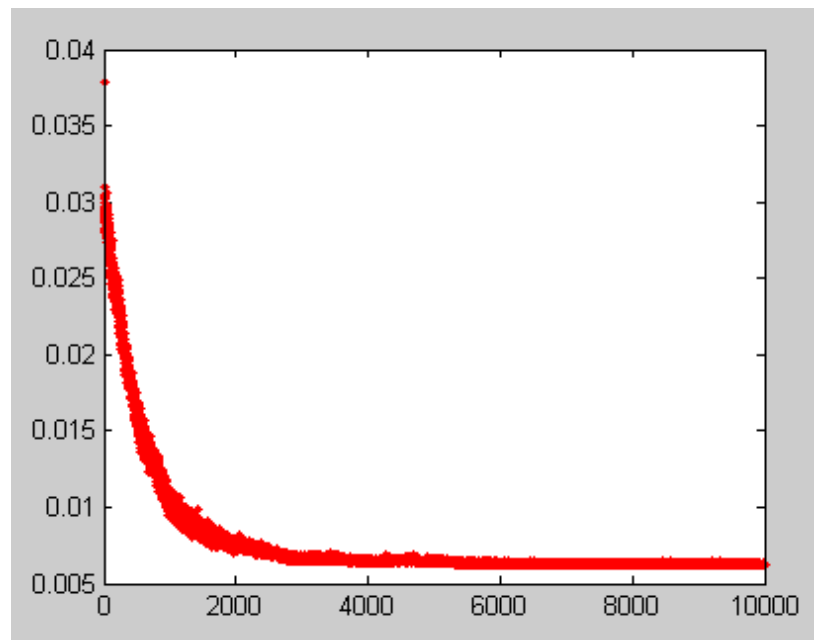

Fig. 5 Optimization process of micro-pits image based on square-alignedarray arrangement

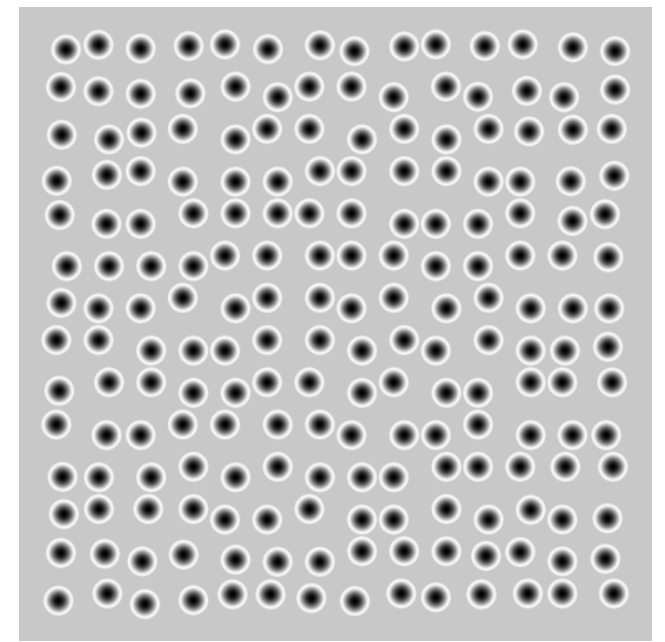

Fig. 6 Micro-pits image of approximate direction-uniformity distribution based on square-aligned-array arrangement

The optimization process is shown in figure 5, in which vertical coordinate represents $S R a_{d c v}$, and the measurement unit is $\mu \mathrm{m}$, while horizontal coordinate represents optimization times. The optimization goal is to make $S R a_{d c v}$ least. The optimization process is convergent. micro-pits image of approximate direction-uniformity distribution is obtained after 10,000 times optimization. The image is shown in figure 6. The $S R a$ is shown in figure 7. The results are: $S R a=3.32 \sim 3.42 \mu \mathrm{m}, S R a_{d c v}=$ $0.62 \%$.

Compared with before, $S R a_{d c v}$ reduces $83.7 \%$. The direction-uniformity of distribution is improved significantly.

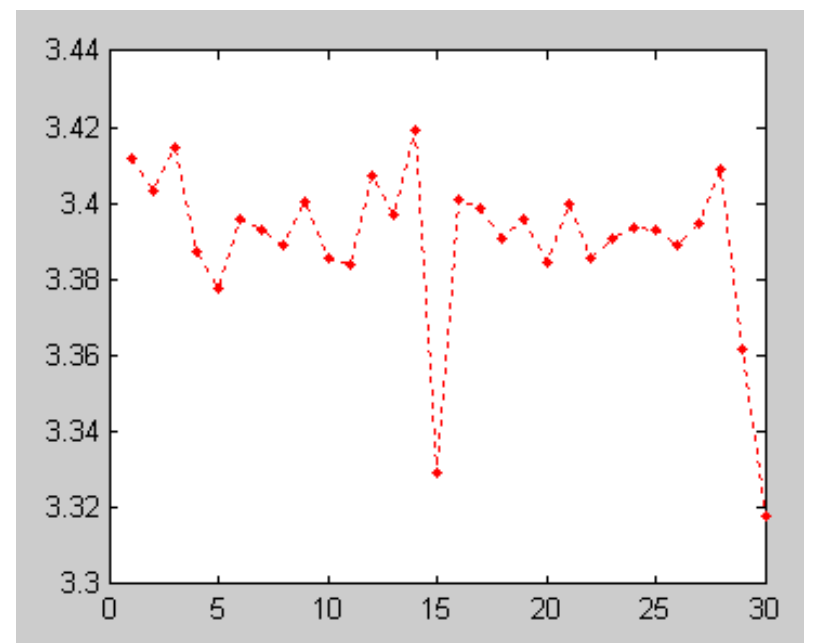

Fig. $7 \mathrm{SRa}$ of micro-pits image of approximate direction-uniformity distribution based on square-aligned-array arrangement

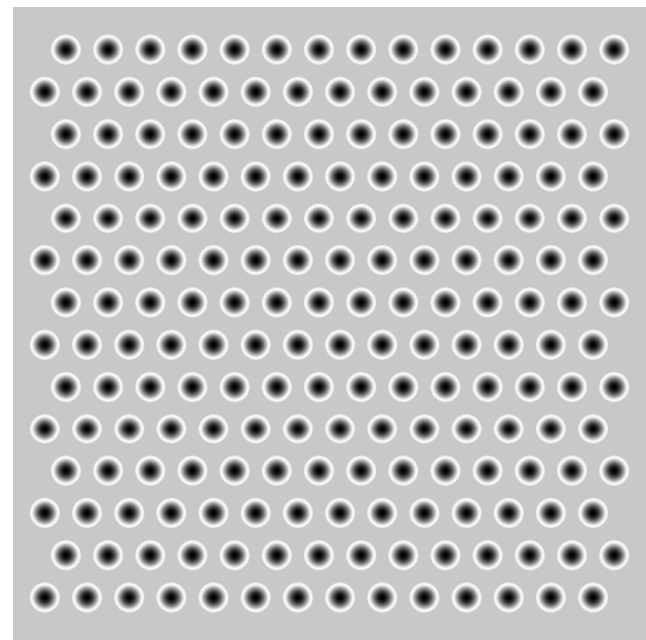

Fig. 8 Regular micro-pits image based on square-staggered-array arrangement 


\section{Partial scrambling micro-pits image generation algorithm based on square-staggered-array arrangement}

Both $S R a$ of micro-pits image based on square-aligned-array arrangement before and after optimization have two minimums in the direction of 90 degrees and 180 degrees, so the value of $S R a_{d c v}$ is correspondingly larger. If the square array is staggered, the minimum in the direction of 90 degrees will be eliminated, therefore the $S R a_{d c v}$ will be reduced furtherly.

Regular micro-pits image. $14 \times 14$ micro-pits square-staggered-array are aligned on the image which size is $3 \times 3 \mathrm{~mm}^{2}$. The distance of the pits is $200 \mu \mathrm{m}$. They are placed in the middle. Figure 8 is the generated regular micro-pits image.

The assessed area is a circle in the middle. The diameter of the circle is $2.35 \mathrm{~mm}$. Figure 9 is $S R a$ of the micro-pits image based on square-staggered-array arrangement, in which vertical coordinate represents $S R a$, and the measurement unit is $\mu \mathrm{m}$, while horizontal coordinate represents direction value, each value represents 6 degrees. The results are: $S R a=2.96 \sim 3.51 \mu \mathrm{m}, S R a_{d c v}=2.93 \%$.

Optimize the micro-pits image. Optimize the image in the same method. The optimization process is shown in figure 10.The optimization process is convergent. Micro-pits image of approximate direction-uniformity distribution is obtained after 10,000 times optimization. The image is shown in figure 11. The $S R a$ is shown in figure 12. The results are: $S R a=3.25 \sim 3.32 \mu \mathrm{m}, S R a_{d c v}=$ $0.32 \%$.

Compared with before, $S R a_{d c v}$ reduces $89.1 \%$. The direction-uniformity of distribution is improved significantly.

Compared micro-pits image of approximate direction-uniformity distribution based on squarestaggered-array arrangement with micro-pits image of approximate direction-uniformity distribution based on square-aligned-array arrangement, $S R a_{d c v}$ reduces $48.4 \%$. The effect is obviously.

\section{Conclusions}

Micro-pits can be uniformly distributed to ensure the area-uniformity of the micro-pits distribution. Micro-pits can be partial scrambled and optimized according to the evaluation standard to ensure the direction-uniformity of the micro-pits distribution. Square partial scrambling micro-pits image generation algorithms based on square-aligned-array arrangement and square-staggered-array arrangement are all effective micro-pits image generation algorithms of uniformity distribution. The latter is more effective.

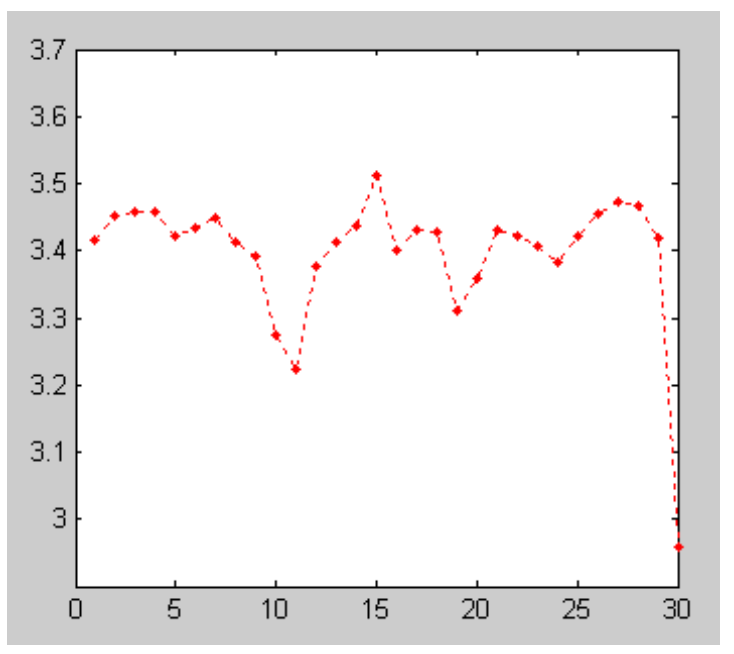

Fig. 9 SRa of the micro-pits image based on square-staggeredarray arrangement

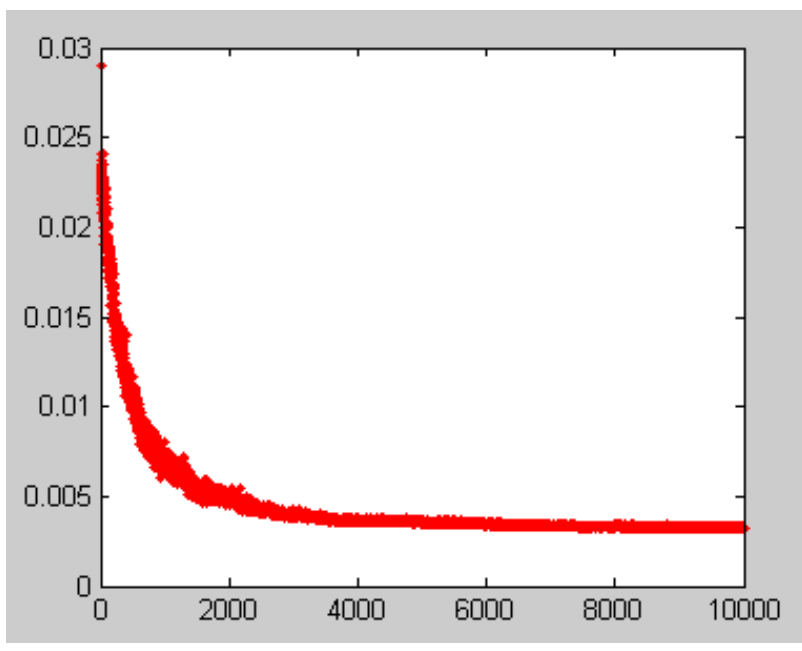

Fig. 10 Optimization process of micro-pits image based on square-staggeredarray arrangement 


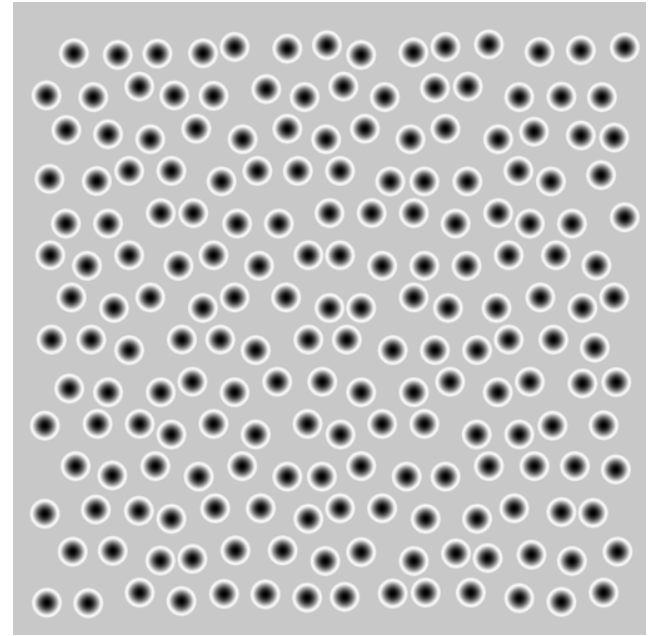

Fig. 11 Micro-pits image of approximate direction-uniformity distribution based on square-staggered-array arrangement

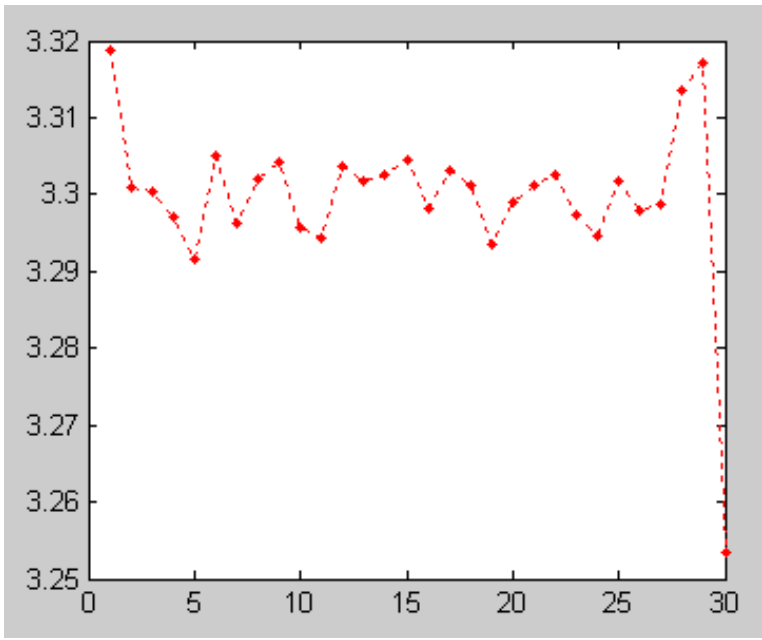

Fig. $12 S R a$ of micro-pits image of approximate direction-uniformity distribution based on square-staggered-array arrangement

\section{References}

[1] Guohui Cao, Wansheng Zhao, Zhenlong Wang. Comparative study of cold roll texturing[J]. ELECTROMACHINING \& MOULD, 2004, (4): 1-5(in Chinese).

[2] Hongcai Wang, Yang Wang, Jian Zhan, et al. The YAG laser texturing technology and application with controllable disorder distribution[C]. 2011 Second International Conference on Mechanic Automation and Control Engineering, 2011, (6): 5458-5462(in Chinese).

[3] Xu Wang, Bing Zhao, Chunfang Liu. Tests research on automobile cold rolled drawing laser texturing steel plate[J]. Automobile Technology \& Material, 1996, (8): 16-20(in Chinese).

[4] Hongcai Wang, Yang Wang. The Height Amplitudes of Surface Roughness based on Area-direction Character and Relative Assessed Indexes[J]. Advanced Material Research, 482-484(2012): 1150-1154.

[5] Changtong Luo. Low dimensional simplex evolution algorithms and their applications[D]. Jilin University, 2007(in Chinese). 
Materials Processing Technology II

10.4028/www.scientific.net/AMR.538-541

The Image Generating Algorithms of Micro-Pits with Direction-Uniformity Distribution of YAG Laser Texturing Roll Based on Square Partial Scrambling 10.4028/www.scientific.net/AMR.538-541.1908 